\title{
The Design of a Dark Field Backscatter Hyperspectral Microscope for Materials Characterization
}

\author{
Marc Walton ${ }^{1}$, Lindsay Oakley ${ }^{1}$, Victoria Cooley $^{2}$ and Danielle Duggins ${ }^{2}$ \\ 1. Northwestern University/Art Institute of Chicago Center for Scientific Studies of the Arts (NU- \\ ACCESS), Evanston, IL, Chicago, USA \\ 2. Northwestern University, Department of Materials Science \& Engineering, Evanston, IL, USA
}

In recent decades, hyperspectral (HS) imaging has become an important molecular characterization tool for cultural heritage. Non-invasive, widefield HS imaging of pigments and binding media on painted surfaces and the identification and monitoring of a variety of other objects, from manuscripts to wood, have been demonstrated[1-3]. When signal is too broad for fingerprinting, often produced from a nonlinear mixed response due to two or more colorants present below the resolution limits of the instrument, HS imaging is usually coupled with complimentary point analysis techniques, such as Raman or FTIR spectroscopy, in order to obtain detailed material information. However, some challenges are encountered with these analytical techniques, notably the contaminating fluorescence background which can swamp the signal in Raman microspectrometry or the low signal-to-noise ratio in FTIR which can necessitate long integration times prohibitive for imaging.

Avoiding these obstacles, a HS imaging technique with high spatial resolution would offer advantages for characterizing at the sub-micron range, with little sample prep or need to access more expensive equipment such as electron microscopy. As of yet, HS microscope configurations are rarely applied outside of the biological sciences. However, a recent dark-field HS optical microscopy experiment demonstrated excellent spatial resolution for the detection of single silver nanoparticles down to $100 \mathrm{~nm}$ diameters using a high numerical aperture objective, indicating the technique's potential for material studies across many disciplines[4].

This work presents the construction and development of a simple dark field backscatter hyperspectral microscope system for the purpose of being able to extract high spatially- and spectrally-resolved information, particularly for pigmented samples. The designed optical path of the microscope is shown in Figure 1. The small angle of illumination for dark field imaging was obtained using an Leitz Wetzlar $5 \mathrm{x}$ dark field objective with a numerical aperture of 0.12. Backscattered light was collected normal to the sample surface. The experiment and instrument design requires a high-intensity light source in the visible/near-IR range. An NKT Photonics SuperK COMPACT white light source coupled through a SuperK SPLIT spectral supercontinuum splitter was used to isolate wavelengths in the VIS/NIR (450$1100 \mathrm{~nm})$ range from the IR (1100-2400nm). The resulting light in the VIS/NIR could be utilized directly for imaging or dispersed in a monochromator (Bentham M300).

Using the monochromator as a wavelength filter ensures that high spectral resolution can be achieved at high spatial resolutions limited solely by the numerical aperture of the objective and the pixel width of the detector. As a range of grating positions and resulting wavelengths were scanned (Figure 1a) and detected using a Resonon Hyperspectral camera, the effective spectral resolution achieved approached that of the camera itself $(\sim 2 \mathrm{~nm})$ as the slit was narrowed as shown in Figure 1b. The theoretical resolution based on the dense diffraction grating (Thorlabs GR50-1205, 1200 lines/mm) and 300mm focal length should be even more precise, approximately $0.4 \mathrm{~nm}$ with power density on the order of tens 
of $\mu \mathrm{W} / \mathrm{nm}$. This high resolution could be leveraged to examine subtle spectral changes resulting from the incorporation of trace impurities or defects. The flexibility and capabilities of the instrument described here is ideal for working with the complex samples often encountered in cultural heritage studies and can be constructed and assembled with modest investment.

\section{References:}

[1] H. Liang, Applied Physics A: Materials Science \& Processing, 106 (2012) 309-323.

[2] G. Agresti, G. Bonifazi, L. Calienno, G. Capobianco, A. Lo Monaco, C. Pelosi, R. Picchio, \& S. Serranti, Journal of Spectroscopy, 2013 (2013) 1-13.

[3] E. Marengo, M. Manfredi, O. Zerbinati, E. Robotti, E. Mazzucco, F. Gosetti, G. Bearman, F. France, \& P. Shor, Analytical Chemistry, 83 (2011) 6609-6618.

[4] P. Z. El-Khoury, A. G. Joly, \& W. P. Hess, Journal of Physical Chemistry C, 120 (2016) 72957298.

[5] The authors gratefully acknowledge support from the Andrew W. Mellon Foundation and the National Science Foundation, Office of International Science \& Engineering (Award \#1743748).

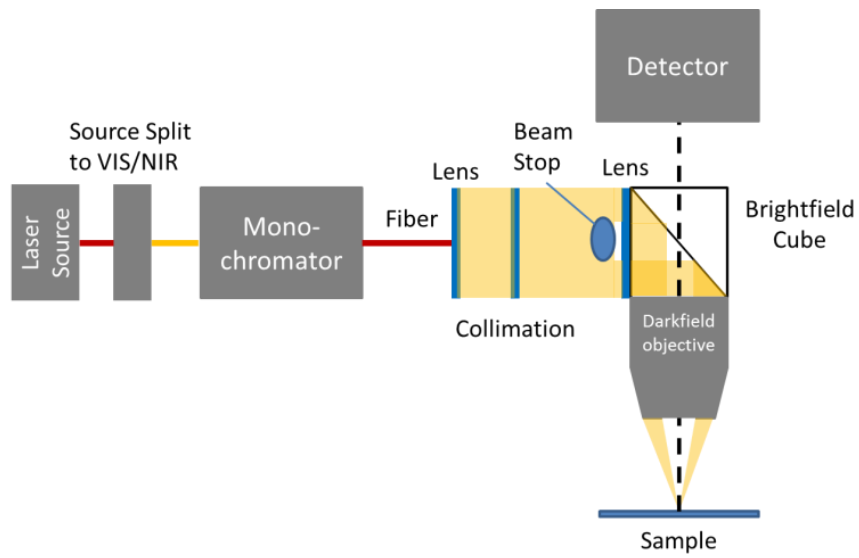

Figure 1. Optical path of the hyperspectral microscope.

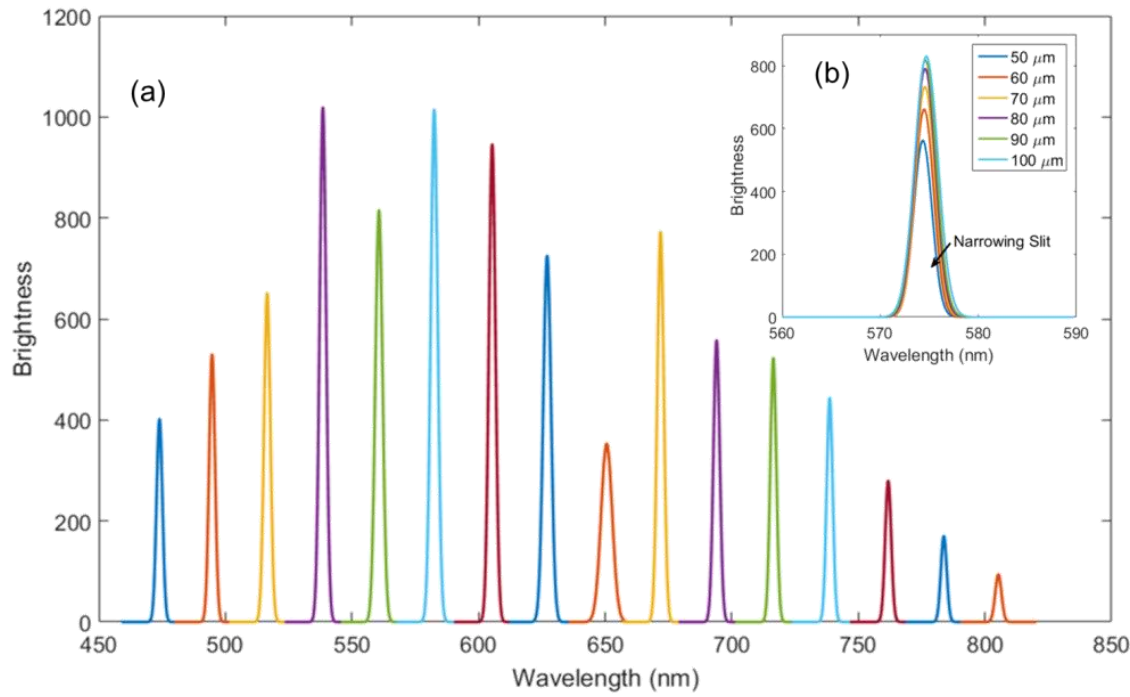

Figure 2. Spectral bands (a) selected from a monochromator to calibrate the diffraction grating position. The inset (b) shows the change in resolution as the slit is narrowed at a constant wavelength. Spectra were averaged over 32,000 pixels acquired in 50 frames with a 3 second integration time. Peaks were fitted with a gaussian curve and the spectral resolution determined from the FWHM. 